\title{
AffectAura: Emotional Wellbeing Reflection System
}

\author{
Daniel McDuff ${ }^{1,2}$, Amy Karlson $^{1}$, Ashish Kapoor ${ }^{1}$, Asta Roseway ${ }^{1}$, Mary Czerwinski ${ }^{1}$ \\ ${ }^{1}$ Microsoft Research, One Microsoft Way, Redmond, USA \\ ${ }^{2}$ MIT Media Lab, 75 Amherst St, Cambridge, USA \\ djmcduff@mit.edu, \{karlson, akapoor, astar, marycz\}@microsoft.com
}

\begin{abstract}
Emotional health is of huge importance to our quality of life. However, monitoring emotional wellbeing is challenging. AffectAura is an emotional prosthetic that allows users to reflect on their emotional states over long periods of time. The system continuously predicts user's valence, arousal and engagement based on information gathered from a multimodal sensor setup. The interface combines these predictions with rich contextual information and allows the user to explore the data. AffectAura has been validated on 100's of hours of data recorded from multiple people who found the system allowed them to reason forward and backward in time about their emotional experiences. This project illustrates the first longitudinally evaluated emotional memory system.
\end{abstract}

\section{Keywords: Emotion; Wellbeing; Visualization; Life logging}

\section{INTRODUCTION}

Emotional health plays a fundamental role in our quality of life [4]. However, monitoring emotional wellbeing is challenging. While we may expect that we are fairly good at assessing our current emotional state, or on average how we have been feeling over the last day or so, do we really accurately assess the highs and lows over the course of a week or month or longer? We conducted a survey that suggested people lacked confidence in their ability of to track their emotional states over weeks or months and many thought an affective life logging tool might be beneficial for reflection [2].

Technology has the ability to offer significant improvement to our emotional wellbeing [1]. One of the aims of affective computing is to use sensors and artificial intelligence in order to recognize emotions. With the large number of ubiquitous sensors available it is becoming increasingly feasible to accurately document our lives and make predictions about users' internal states. However, open questions remain about how to make this information understandable for users and how useful it is for reflection. We present AffectAura, the first longitudinally evaluated system for emotion reflections that represents a milestone in answering these questions. AffectAura combines a multi-modal sensor setup for life logging, an affect prediction engine for continuous prediction of user's valence, arousal and engagement and an interface combining affective predictions with rich contextual information.

This system has similarities with the Affective Diary [3]. However, there are important differences. The Affective Diary enables semi-automated diary entry creation, combining hand written entries with abstract representation of mood and activity. The tool was designed for the active creation of diary entries and to investigate the impact of affective information on creativity. AffectAura is a completely automated system for exploring the potential reflective power of pairing contextual and affective information.

\section{LIFE LOGGING}

\section{A. Sensor System}

A multimodal life logging sensor system collects signals with affective information. Figure 1a shows the sensor configuration at a user's workstation. The following sensors are used:

Webcam: An active appearance model (AAM) tracks the head
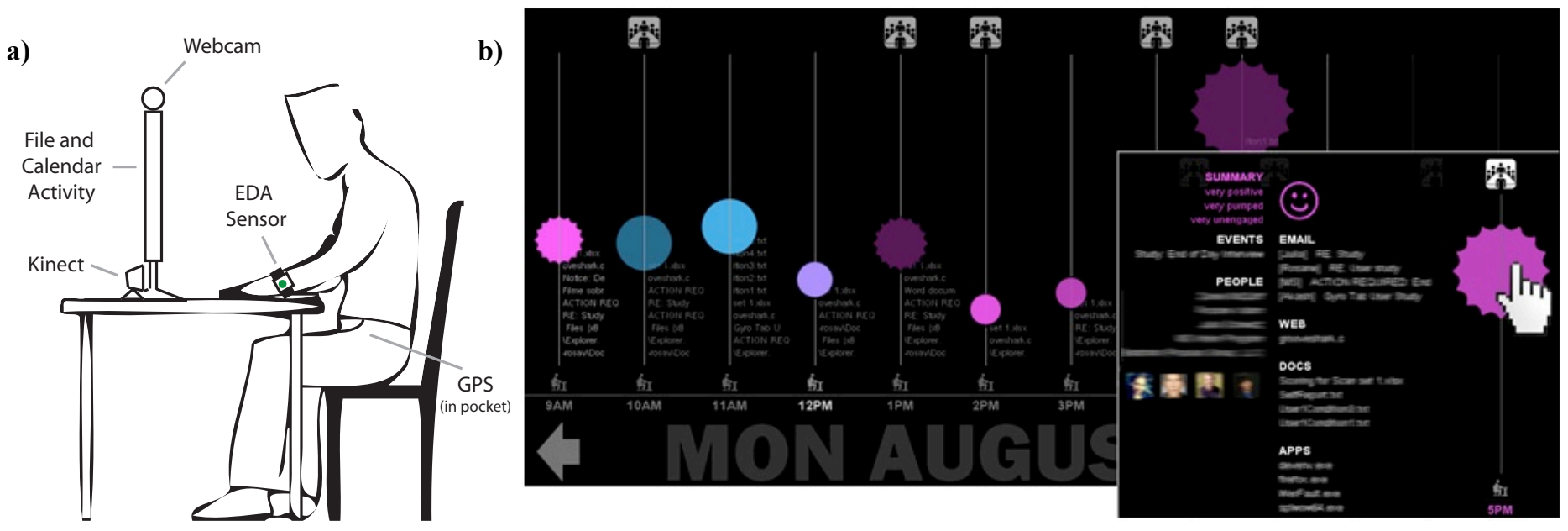

Figure 1. a) Life logging sensor configuration, b) an example of the AffectAura interface. Detailed explorations of an hour (5pm) enabled by hovering over the bubble (inset). 
pitch, jaw and roll from a webcam stream. The webcam is mounted on the participants' computer screen.

Kinect: Posture (direction of lean: left-to-right and forward-toback) is determined using depth information and skeleton tracking using a Kinect device.

Microphone: Voice activity and speech features are extracted from a WAV file recorded using a mic in the Kinect device.

Electrodermal Activity: Electrodermal activity (EDA) and wrist motion features are extracted from a wrist worn sensor.

GPS: A GPS device records latitude and longitude data.

File Activity: A custom logging application records file activity at the user's desktop, including: web URL's visited, documents opened, applications used, and emails sent/received.

Calendar Scraper: A custom calendar scraper records time and attendees of meetings scheduled in the user's calendar.

\section{B. Affective Prediction Engine}

To surface affective signals from the multi-modal sensor information a custom affect prediction engine is used. To build the engine training data and self-report labels were collected from five participants over two days each.

The system uses Neighborhood Component Analysis (NCA) to reduce the dimensionality of the input data whilst maximizing the distance between examples with different labels and minimizing the distance between examples with the same labels. A nearest neighbor classifier is used to predict labels for new examples. The details of the affect prediction engine are described in more depth in [2].

\section{AFFECTAURA's INTERFACE}

AffectAura is an interactive visualization of the user's predicted affective states over time. A screenshot of the interface is shown in Figure 1b. AffectAura consists of an "affect timeline" capturing the ebb and flow of affect represented by a series of bubbles. The user can drill down by hovering over a bubble to reveal a detailed breakdown of their activities and interactions associated with that time.

Valence of the user is represented by the color of the bubble. Pink, purple and blue represent positive, neutral and negative affect, respectively.

Arousal of the user is represented by the shape of the bubble. A circle or a "burst" were chosen to represent calm and "pumped up" respectively.

Engagement of the user is represented by the opacity, with higher opacity corresponding to greater engagement.

For each hour, the user's desktop activity (file and application activations) is represented by the radius and height of each bubble. A subset of the activities themselves are listed as a ghosted column of text to lend a sense of amount of file and application interaction that occurred. An icon displayed at the top of the column represents meetings scheduled in the user's digital calendar during a particular hour. Similarly, locations (based on GPS and file activity information) are represented as an icon at the bottom of the column.

To allow for exploration of the events of each hour, the user could hover over any bubble to reveal details of their activities logged during the hour. (Figure 1b, inset). The artifacts presented on hover included calendar events, people (names and when available, images), email titles (with sender name), website URLs, document names, and applications used.

\section{INTERACTION}

Users will be able to interact with the AffectAura interface and view the affective and contextual information collected over many days during a user study. They will be able to evaluate the user experience offered by the "affect timeline" and the utility of representing automatically collected affective information for reflective purposes. The interface will be available on a tablet device and/or computer.

\section{VALIDATION AND CONCLUSIONS}

AffectAura has been evaluated in a user study. The interface was demonstrated to allow users to leverage cues from AffectAura to construct stories about their days, even after they had forgotten particular incidents or their related emotional tones. Six participants ( 3 females) took part in the evaluation of AffectAura. On average, participants recorded over 10 hours of sensed data per day, totaling over 240 hours of logged data. Five of the six participants told us that affect would be useful for reflecting on events collected in the user interface.

This project illustrates the first longitudinally evaluated emotional memory system. AffectAura incorporates a multimodal sensor set-up for continuous life logging, an affect prediction engine for continuously predicting valence, arousal and engagement states of the user. The interface correlates this with information on events, communications and data interactions. Users' can interact with AffectAura's interface and evaluate the utility of an emotional reflection tool.

\section{REFERENCES}

[1] D. Coyle, C. Linehan, K. P. Tang, and S. Lindley, "Interaction Design and Emotional Wellbeing," Workshop Abstract, Proceedings of the 2012 annual conference on Human factors in computing systems, May 2012.

[2] D. McDuff, A. Karlson, A. Kapoor, A. Roseway and M. Czerwinski, "AffectAura: An Intelligent System for Emotional Memory," Proceedings of the 2012 annual conference on Human factors in computing systems, May 2012.

[3] A. Ståhl, K. Höök, M. Svensson, A. Taylora and M. Combetto, "Experiencing the Affective Diary," Pers Ubiquit Comput, 365-378, 2009.

[4] WHO. Mental Health Action Plan for Europe: Facing the Challenges, Building Solutions In proc WHO European Ministerial Conference on Mental Health 2005. 